\title{
Das Ökologieskript der Europäischen Union und seine Akzeptanz in den Mitglieds- und Beitrittsländern der EU
}

Jürgen Gerhards E Holger Lengfeld

Berliner Studien zur Soziologie Europas

Nr. 1

September 2005

Freie Universität Berlin, Institut für Soziologie, Garystraße 55, D-14195 Berlin 
Die „Berliner Studien zur Soziologie Europas“ des Lehrstuhls für Makrosoziologie der Freien Universität Berlin verstehen sich als ein Ort zur Vorpublikation von Beiträgen, die später in Fachzeitschriften und Sammelbänden veröffentlicht werden sollen. Die Beiträge sollen helfen, eine Soziologie Europas zu profilieren; sie stehen auch im Kontext des Master-Studiengangs „Soziologie - Europäische Gesellschaften“.

Gegenstand der Reihe sind Beiträge zur Analyse der Herausbildung einer europäischen Gesellschaftsstruktur und -kultur, vergleichende Analysen, die die Unterschiede und Gemeinsamkeiten zwischen verschiedenen europäischen Gesellschaften thematisieren, sowie theoretische Versuche einer Soziologie Europas.

Ziel der Reihe ist es, durch die frühe Verbreitung dieser Arbeiten den wissenschaftlichen Gedankenaustausch zu fördern. Die Beiträge sind nur über das Internet als pdf-Datei zu beziehen.

Zitationsweise: BSSE-Arbeitspapier Nr.1. Berlin: Freie Universität Berlin.

Dieser Artikel erschien auch in: Zeitschrift für Soziologie 35 (2006) S. 24-40. 


\begin{abstract}
Der Prozess der zunehmenden europäischen Integration lässt sich nicht nur als ein Prozess der Zunahme europäischer Regelungen und der Ausbildung eines eigenständigen politischen Herrschaftsverbandes, sondern aus soziologischer Perspektive auch als ein Prozess der Etablierung einer europäischen Gesellschafts- und Werteordnung begreifen. In diesem Beitrag wird gefragt, in welchem Maße die Bürgerinnen und Bürger der Europäischen Union und der gegenwärtigen Beitrittskandidaten die institutionalisierten Ökologiewerte der EU unterstützen. Zunächst wird das Ökologieskript der EU unter Rückgriff auf die Entwicklung des Primär- und Sekundärrechts rekonstruiert. Anschließend untersuchen wir anhand von Daten des „Gallup Millennium Survey“ 1999 und des ISSP 2000, in welchem Maße die Ökologievorstellungen der EU von den Bürgern in den Mitglieds- und Beitrittsländern geteilt werden und welche Ursachen für auftretende Werteunterschiede verantwortlich sind. Die Ergebnisse deuten darauf hin, dass die mittelosteuropäischen Länder und die Beitrittskandidaten mittelfristig kaum weitergehende EU-Initiativen zum Umweltschutz unterstützen werden. Langfristig jedoch kann die Bereitschaft zum Umweltschutz wieder ansteigen, wenn die erwartete ökonomische Modernisierung der neuen Mitgliedstaaten voranschreitet, und wenn sich in ihrem Gefolge die grundlegenden Werthaltungen vom Materialismus hin zum Postmaterialismus ändern.
\end{abstract}




\section{Einleitung 1}

Aus politikwissenschaftlicher Perspektive ist die Europäische Union ein supranationaler politischer Herrschaftsverband, der zwischen den beiden Idealtypen Bundesstaat einerseits und Staatenbund andererseits zu verorten ist (vgl. statt anderer Kohler-Koch et al. 2004; Weidenfeld 2004). Bekanntlich haben die Nationalstaaten einen erheblichen Teil ihrer Souveränität auf die EU übertragen, ihre Bürger sind den Beschlüssen der EU unmittelbar unterworfen, Europarecht bricht nationales Recht, die Kommission überwacht die Implementierung der Beschlüsse, und der europäische Gerichtshof kann die Mitgliedstaaten bei Nichtbefolgung sanktionieren (Lepsius 1990). Die Verlagerung von Souveränitätsrechten von den Nationalstaaten auf die EU manifestiert sich in einer Vielzahl von Indikatoren. So ist die Summe von Entscheidungen seitens des Europäischen Rates bzw. der Europäischen Kommission kontinuierlich gestiegen, die Anzahl der Fachministerräte ebenfalls, die Verflechtung zwischen europäischer und nationaler Politik hat zugenommen (Knill 2001), und auch die intermediären Organisationen und Interessengruppen richten ihr Augenmerk zunehmend auf die europäische Ebene (Fligstein/Stone Sweet 2002; Roose 2003; Stone Sweet et al. 2001; Wessels 1997: 283).

Der Prozess der zunehmenden europäischen Integration lässt sich aber nicht nur als ein Prozess der Zunahme europäischer Regelungen und der Ausbildung eines eigenständigen politischen Herrschaftsverbandes beschreiben, sondern aus soziologischer Perspektive auch als ein Prozess der Etablierung einer europäischen Gesellschafts- und Werteordnung. Man kann die Europäische Union nämlich als Werteunternehmer interpretieren, der sehr dezidierte Vorstellungen von einer europäischen Gesellschaft entwickelt hat. Die EU verfügt über ein Skript einer idealen Gesellschaft, das sie mit ihren "policies" auch zu realisieren versucht. Dieses Skript, das sich im EU-Primär- und Sekundärrecht und dem Verfassungsentwurf manifestiert, soll den Mitgliedstaaten wie den Institutionen der EU als normative Richtschnur des eigenen Handelns dienen.

Wir haben an anderer Stelle genauer beschrieben, wie dieses Skript einer europäischen Gesellschaft aussieht und dabei unterschiedliche Wertsphären unterschieden (Religion, Familie und Geschlechterrollen, Demokratie und Zivilgesellschaft, Wirtschaft; vgl. Gerhards/Hölscher 2005). In diesem Beitrag knüpfen wir an diese Analysen an. Teil des Gesellschaftsskripts der EU ist auch eine spezifische Vorstellung des Umgangs der Menschen mit der natürlichen Umwelt. Bei der Gründung der Europäischen Wirtschaftsgemeinschaft in den 1950er Jahren noch vollkommen bedeutungslos, hat die EU-Umweltpolitik mittlerweile einen beachtlichen Bedeutungszuwachs erfahren (Bailey 2003; Barnes/Barnes 1999; McCormick 1999): Der Umfang des um-

1 Wir bedanken uns bei David Glowsky und Juliane Seifert, die die Materialen zur Rekonstruktion der EU-Vorstellungen von Ökologie zusammengestellt haben, sowie bei Denis Huschka, Jochen Roose und Mike Steffen Schäfer für konstruktive Kritik. 
weltpolitischen Regelwerks hat stark zugenommen, und der Umweltschutz ist in den Zielkanon des Verfassungsentwurfs aufgenommen worden. Umweltschutz rangiert damit auf gleicher Höhe wie das Prinzip der Freizügigkeit, die soziale Marktwirtschaft und die Gleichberechtigung der Geschlechter.

In einem ersten Schritt rekonstruieren wir die Genese und den Gehalt der Ökologievorstellungen der europäischen Union als Bestandteil des Gesellschaftsskripts der EU. Dazu werfen wir einen Blick auf die historische Entwicklung des EU-Rechts seit den 1950er Jahren bis zur jüngsten Osterweiterung im Jahre 2004. In einem zweiten Schritt prüfen wir, ob und in welchem Maße die Ökologievorstellungen der EU von den Bürgern in den Mitglieds- und Beitrittsländern (Rumänien, Bulgarien und Türkei) unterstützt werden. Wir fragen, ob und in welchem Ausmaß es zwischen den europäischen Gesellschaften Einstellungsunterschiede im Hinblick auf die Unterstützung der Ökologievorstellungen der EU gibt. Zur Beschreibung dieser Einstellungen verwenden wir Daten des "Gallup International Millennium Survey“ aus dem Jahr 1999.

Dabei gehen wir von der Annahme aus, dass die Unterstützung von im Recht institutionalisierten Werten durch die Bürger nicht unerheblich für die Legitimität der EU-Politiken ist. In Wettbewerbsdemokratien werden Eliten nicht dauerhaft gegen die Präferenzen ihrer Bürger handeln. Die Konkurrenz der Eliten um Regierungspositionen und ihre Abhängigkeit von den Willensäußerungen der Bürger macht es sehr wahrscheinlich, dass sich die Eliten an den Wünschen der Bürger orientieren und sich im Hinblick auf diese Wünsche als responsiv erweisen. Insofern können die Wertüberzeugungen der Bürger unter demokratischen Strukturbedingungen - vielfach über Prozesse der Interessensartikulation und -aggregation vermittelt - zur Strukturveränderung von Gesellschaften beitragen (vgl. Almond et al. 2003: 42; Page/Shapiro 1983). Für das europäische Mehrebenensystem bedeutet dies, dass je unterschiedlicher die Wertorientierungen zur Umwelt zwischen den Mitgliedstaaten sind, desto geringer ist die Wahrscheinlichkeit einer europäisch koordinierten Umweltpolitik.

Die deskriptiven Befunde werden zeigen, dass das Umweltskript der EU bei den Bürgern Europas durchaus unterschiedliche Unterstützung erfährt. Um Hinweise auf die langfristige Persistenz bzw. Wandlungsfähigkeit dieser nationalen Einstellungsdifferenzen zu erhalten, versuchen wir im dritten Schritt, die Unterschiede in den Einstellungen der nationalen Bevölkerungen zu erklären (Abschnitt 4). Dazu formulieren wir sechs Hypothesen, die wir anschließend multivariat überprüfen. Da im Rahmen des „Gallup International Millennium Survey“ kaum Informationen über die sozio-ökonomische Lage der Befragten erhoben wurden, verwenden wir für die Kausalanalyse Daten des "International Social Science Programme“ (ISSP) aus dem Jahr 2000. Welche Schlussfolgerung man aus diesen Befunden für die Zukunft der EU-Umweltpolitik ziehen kann, diskutieren wir im letzten Abschnitt. 


\section{Das Ökologieskript der EU}

Die EU-Umweltpolitik hat seit den 1970er Jahren eine erstaunliche Entwicklung genommen. Zum Zeitpunkt des „Take Off“ der EU im Jahre 1957 war keinerlei Zuständigkeit der neuen Institutionen für den Schutz der natürlichen Umwelt vorgesehen. Bis Anfang der 1970er hatte es nur vereinzelte Umweltrichtlinien gegeben, die aber auf Grund ihrer geringen Bedeutung nicht als europäische Umweltpolitik verstanden werden können (Fürst 2004: 42). Dies änderte sich im Laufe der 1970er Jahre. Seitdem hat das Thema Ökologie zunehmend an europäischer Relevanz gewonnen. Den Prozess der Bedeutungszunahme des Umweltthemas kann man in drei Phasen unterteilen.

\subsection{Die umweltpolitische Startphase}

Im Anschluss an die erste Umweltschutzkonferenz der Vereinten Nationen in Stockholm 1972 verabschiedeten die Staats- und Regierungschefs der damaligen EG eine Erklärung zur Umwelt- und Verbraucherpolitik. In dieser Erklärung wurde die Kommission beauftragt, ein Aktionsprogramm für Umweltschutz auszuarbeiten. Anders als Verordnungen und Richtlinien besitzen Aktionsprogramme keine Gesetzeskraft. Gleichwohl ist ihre Bedeutung nicht zu unterschätzen, da sie dem gesetzgeberischen Produktionsprozess als Richtschnur für konkrete politische Initiativen dienen (vgl. Barnes/Barnes 1999; Krämer 1992).

1973 beschloss die Kommission das erste umweltpolitische Aktionsprogramm. Darin legte sie die Grundsätze der Umweltpolitik fest, so unter anderem das Präventions- und das Verursacherprinzip. In den weiteren fünf Aktionsprogrammen wurden die umweltpolitischen Zielsetzungen mehr und mehr konkretisiert. Seit dem dritten Aktionsprogramm (1982-1986) wurde die Integration der Umweltpolitik in andere Politikbereiche festgeschrieben. Im fünften (1993-2000) und sechsten (20012001) Aktionsprogramm schließlich wurden nicht nur konkrete Umweltprobleme und deren Lösung durch Politiken behandelt, sondern auch Prinzipien der Kooperation der EU-Akteure mit außerstaatlichen Akteursgruppen definiert.

Da der Umweltschutz nicht Teil der römischen Verträge und damit nicht als europäische Aufgabe im Primärrecht definiert war, besaß die Kommission für ökologische Politiken keine Legitimation. Dieses Legitimationsdefizit wurde, wie häufig bei der Ausdehnung der Herrschaftsbereiche der EU, durch die Strategie des "Scriptbridging" überwunden. ${ }^{2}$ Bekanntlich ist die EU vor allem und in erster Linie als Wirtschaftsgemeinschaft entstanden. Die Ausdehnung der Zuständigkeiten auf andere Politikfelder erfolgte zumeist durch die interpretatorische Verbindung mit dem

2 Der Begriff schließt an das Konzept des „Frame-bridging“ an, wie es vor allem von David Snow in der Bewegungsforschung entwickelt wurde (vgl. Snow et. al. 1986; Snow/Benford 1988). 
Wirtschaftsskript. ${ }^{3}$ Mit dieser Strategie hat die EU auch ihre Zuständigkeit auf ökologische Fragen ausgedehnt (vgl. Knill 2003: 19 ff.; Johnson/Corcelle 1989: 2 ff.). Laut Präambel der Römischen Verträge ist das Ziel der EU die Verbesserung der Lebensund Beschäftigungsbedingungen. In Artikel 2 des EG-Gründungsvertrages wird ihr die Aufgabe zugeschrieben, eine harmonische Entwicklung des Wirtschaftslebens zu fördern. Auch wenn die Gründerväter bei dem Terminus „Lebensbedingungen“ vor allem die ökonomischen Lebensbedingungen vor Augen hatten, ermöglicht der Begriff im Grundsatz, auch ökologische Lebensbedingungen als relevante Aufgabe einzuschließen.

Im Vertragstext selbst wurde Umweltschutz jedoch nicht explizit erwähnt. Insofern blieben die umweltpolitischen Maßnahmen der EU bis zur Einheitlichen Europäischen Akte (EEA) 1987 in erster Linie ökonomisch legitimiert. Von 1972 bis 1987 basierten daher alle umweltpolitischen Maßnahmen auf wirtschaftspolitischer Legitimationsgrundlage (Fürst 2004: 42; Knill 2003: 22). ${ }^{4}$ Trotz der dünnen Rechtslage verselbstständigte sich die Umweltpolitik und löste sich von anderen Bereichen ab. Neben den Aktionsprogrammen wurden im Laufe der Zeit rund 200 verbindliche Rechtsakte verabschiedet. Der Europäische Gerichtshof (EuGH) hat diese Auslegung des EU-Rechts legitimiert, indem er z. B. den Erlass von einheitlichen Produktionsvorschriften akzeptierte. Durch Produktionsvorschriften werden z.B. Emissionswerte festgelegt und damit Umweltpolitik betrieben. ${ }^{5}$

3 Für die Bereiche Familie und Geschlechterbeziehungen haben wir das an anderer Stelle genauer analysiert (vgl. Gerhards/Hölscher 2003). Die EU betreibt an jenen Stellen Familienpolitik, wo Familienfragen Wirtschaftsfragen tangieren. Da die politische Regulierung des Verhältnisses von Wirtschaft und Familie auf das Binnenleben von Familien zurückwirkt, betreibt die EU auf mittelbarem Wege auch Familienpolitik und versucht ihre Vorstellungen einer wünschenswerten Familie zu etablieren.

4 Neben Artikel 2 wurde auch Artikel 94 des EGV angewendet: „Der Rat erlässt einstimmig auf Vorschlag der Kommission und nach Anhörung des Europäischen Parlaments und des Wirtschaftsund Sozialausschusses Richtlinien für die Angleichung derjenigen Rechts- und Verwaltungsvorschriften der Mitgliedstaaten, die sich unmittelbar auf die Errichtung oder das Funktionieren des Gemeinsamen Marktes auswirken."

5 Knill (2003: 25 f.) nennt drei Gründe, warum es zur Entstehung eines eigenständigen europäischen Politikfeldes „Umweltpolitik“ gekommen ist: (1) Erhöhter ökologischer Problemdruck in den Mitgliedstaaten vor allem zu Beginn der 1980er Jahre (z. B. die Diskussion um das Waldsterben in Deutschland); (2) Vorreiter erhöhten die Dynamik (aufgrund des grenzüberschreitenden Charakters des ökologischen Problems haben einige Mitgliedstaaten wie Deutschland, Niederlande und Dänemark - unterstützt vom EuGH - versucht, durch europäische Maßnahmen den Druck auf andere Länder zu erhöhen); (3) Unterschätzung der Wirkung der EU-Vorschriften (in den Verhandlungen unterschätzen einige Mitgliedstaaten die konkreten finanziellen Konsequenzen der EUVorschriften; so stimmten sie z. B. strengen Richtlinien für die Trinkwasserqualität zu, ohne sich über die langfristigen Kosten der Umsetzung im Klaren zu sein). 


\subsection{Die Phase der rechtlichen Etablierung}

Zum 1. Juli 1987 wurde der EWG-Vertrag um die Einheitliche Europäische Akte (EEA) erweitert. In ihr wurde die Umweltpolitik als separates Politikfeld von anderen Gebieten abgelöst und erhielt eine eigene Generaldirektion, wodurch die institutionelle Stellung der Umweltpolitik unterstrichen wurde. Durch die Verträge von Amsterdam und Maastricht wurde die Ausdifferenzierung eines eigenen Politikfeldes für Umweltpolitik bekräftigt. 1994 wurde die Europäische Umweltagentur eröffnet, die in Kopenhagen Umweltdaten sammelt und für Brüsseler Entscheidungen aufbereitet. Den symbolisch wichtigsten Höhepunkt dieser Entwicklung markiert die Aufnahme des Umweltschutzes in die Präambel des Entwurfs der Europäischen Verfassung. In Artikel II (3) heißt es: „Die Union wirkt auf die nachhaltige Entwicklung Europas auf der Grundlage eines ausgewogenen Wirtschaftswachstums und von Preisstabilität, eine in hohem Maße wettbewerbsfähige soziale Marktwirtschaft, die auf Vollbeschäftigung und sozialen Fortschritt abzielt, sowie ein hohes Maß an Umweltschutz und Verbesserung der Umweltqualität hin“. Die näheren Ziele werden in Art. III-233 definiert. Dazu zählen im Einzelnen:

- Erhaltung und Schutz der Umwelt sowie Verbesserung ihrer Qualität,

- Schutz der menschlichen Gesundheit,

- umsichtige und rationelle Verwendung der natürlichen Ressourcen,

- Förderung von Maßnahmen auf internationaler Ebene zur Bewältigung regionaler oder globaler Umweltprobleme.

Innerhalb von 20 Jahren ist die Idee einer zu schützenden Umwelt zum festen Bestandteil des Gesellschaftsskripts der EU geworden. Dies bedeutet natürlich nicht, dass das primäre Ziel der EU, ein wirtschaftlich integriertes Europa zu erreichen, durch die Idee eines ökologischen Europas ersetzt wurde. Gleichwohl wurden ökonomische Rationalitätskriterien zunehmend durch ökologische Kriterien ergänzt, die den ökonomischen Kriterien zum Teil klar widersprechen. Dabei blieb die ökologische Idee keine abstrakte Ideologie, sondern war und ist folgenreich und wirkmächtig für eine Vielzahl konkreter Entscheidungen. ${ }^{6}$

6 Dies sei an einem Beispiel illustriert. Der Europäische Gerichtshof (EuGH) hat am 17.09.2002 in einem Urteil bestätigt, dass anspruchsvolle Umweltschutzkriterien, die über die derzeitigen gesetzlichen Anforderungen hinausgehen, bei der wettbewerblichen Vergabe von Leistungen des öffentlichen Personennahverkehrs (ÖPNV) berücksichtigt werden dürfen. Dies ist laut EuGH auch dann erlaubt, wenn nur wenige Verkehrsunternehmen diese Umweltkriterien erfüllen können. So hatte die Stadt Helsinki 1997 in einer Ausschreibung für den innerstädtischen Busverkehr als Zuschlagskriterium u. a. Emissionsstandards festgelegt, die nur von Erdgasbussen eingehalten werden können. Nachdem das gemeindeeigene Unternehmen den Zuschlag erhielt, klagte ein Konkurrent, der darin eine unrechtmäßige Diskriminierung sah. Der zuständige Generalanwalt beim EuGH hatte argumentiert, dass eine Gemeinde als öffentlicher Aufgabenträger im Rahmen ihrer Verantwor- 


\subsection{Die Erweiterungsphase}

Die EU hat ihr Ökologieskript mit dem Beitritt der zehn mittelosteuropäischen Staaten im Jahre 2004 auch auf die neuen Länder ausgedehnt und diese der europäischen Werteordnung unterworfen. Die Kopenhagener Kriterien von 1993 verlangen u. a., dass jedes Beitrittsland den gemeinschaftlichen Besitzstand (Acquis Communautaire) in seine nationalen Rechtsvorschriften aufnimmt. Das von den Beitrittsländern zu übernehmende Gemeinschaftsrecht umfasst insgesamt 31 Kapitel, von denen sich eines mit dem Umweltschutz befasst (Kapitel 22). Dieses Umweltschutzkapitel ist selbst wiederum in elf verschiedene Bereiche gegliedert. Diese bilden die Grundlage für die Überprüfung der Anpassungsfortschritte (für die Kriterien im Einzelnen vgl. Anhang 1). ${ }^{7}$

Beabsichtigt ist, dass die formale rechtliche Angleichung mit der praktischen Umsetzung der Rechtsvorschriften einhergeht. So sollen Umweltschutzförderprogramme in die Wirtschaftspolitik und andere Politikbereiche des jeweiligen Landes integriert werden. Außerdem soll der ökologische Vorteil der biologischen Vielfalt erhalten bleiben. Die Bewerberländer müssen „realistische langfristige nationale Strategien zur allmählichen effektiven Angleichung" (Europäische Gemeinschaften 19952005a: 1) aufstellen und umgehend mit deren Umsetzung beginnen. Welche Umweltbereiche vorrangig zu behandeln sind, wurde von der Kommission für jedes Land separat festgelegt. Besonderen Nachholbedarf sieht die Kommission in den Bereichen Luft- und Wasserverschmutzung sowie in der Abfallentsorgung (Europäische Gemeinschaften 1995-2005a: 2).

Die Angleichung ist für die Beitrittsländer mit erheblichen finanziellen Kosten verbunden. Geschätzt wird, dass die notwendigen Investitionskosten etwa 100 bis 120 Milliarden Euro betragen (Roth-Behrendt/Nowak 2004: 318). Dabei handelt es sich vor allem um Infrastrukturkosten für Trinkwasserversorgung, Abwasserbehandlung und Abfallentsorgung. Im Grundsatz müssen die Beitrittsländer die erforderlichen Mittel selbst bereitstellen. Dabei werden sie jedoch durch verschiedene bilaterale Programme der Gemeinschaft unterstützt. Diese Unterstützung hat bereits vor dem Beitritt der neuen Mitgliedstaaten in großem Umfang stattgefunden. Das

tung umweltpolitische Zielvorstellungen festlegen und diese auch in Bestbieterkriterien für die Ausschreibung festschreiben darf: Die Einhaltung auch sehr anspruchsvoller Abgasgrenzwerte im ÖPNV gehöre zu jenen Bestbieterkriterien, die von der Dienstleistungsrichtlinie (RL 92/50) vorgesehen seien und könne nicht als Diskriminierung anderer Bieter interpretiert werden, deren Fahrzeuge solche Grenzwerte nicht erreichen (Nähere Informationen finden sich unter http://www.bmu.de/pressearchiv/14_legislaturperiode/pm/1758.php; für die ausführliche Urteilsbegründung siehe http://www.ihk-niederrhein.de/downloads/EuGH-oeffentliche_Ausschreibungen_Umweltkriterien.pdf.).

7 Im Rahmen der Beitritt-I-Verhandlungen wurden jedoch insgesamt 60 Übergangsfristen für die Anwendung des Gemeinschaftsrechts vereinbart, insbesondere in den kostenintensiven Bereichen der Abwasserentsorgung und der Luftreinhaltung (BMU 2005). 
zentrale EU-Instrument ist das 1989 ursprünglich für Polen und Ungarn konzipierte PHARE-Programm (Pologne, Hongrie Assistance à la Reconstruction Economique). PHARE stellt den Beitrittsländern seit 2000 jährlich rund 1,5 Milliarden Euro bereit. Ebenfalls seit 2000 wurden im Rahmen des ISPA-Programms (Instrument for Structural Policies for Pre-Accession) zusätzliche Mittel in jährlicher Höhe von einer Milliarde Euro zur Bekämpfung der Probleme im Umweltbereich reserviert. Die Inanspruchnahme der Mittel ist davon abhängig, dass alle neuen Investitionen der Beitrittsländer mit dem Acquis vereinbar sind. Darüber hinaus versucht die EU, internationale Finanzinstitute dahingehend zu beeinflussen, entsprechende Darlehen von der Konformität mit dem Umwelt-Acquis abhängig zu machen (Europäische Gemeinschaften 1995-2005a: 3).

Fassen wir unsere Rekonstruktion des EU-Ökologieskripts zusammen. Der Schutz der natürlichen Umwelt hat im Gesellschaftsskript der EU im Zeitverlauf zunehmend an Bedeutung gewonnen und nimmt heute einen beachtlichen Stellenwert ein. Auch wenn die EU weiterhin in erster Linie eine Wirtschaftsgemeinschaft ist, ist die Institutionalisierung ökonomischer Rationalitätskriterien durch ökologische Kriterien ergänzt worden. Die EU hat sich die Umweltpolitik eigeninitiativ, durch die Hintertür der Harmonisierung des gemeinsamen Marktes, angeeignet und Schritt für Schritt im Primärrecht der Verträge verankert. Weiterhin hat sie ihre umweltpolitischen Zielsetzungen über die Aktionsprogramme kontinuierlich ausgebaut. Schließlich hat sie den Beitritt der mittelosteuropäischen Länder daran geknüpft, dass diese umfangreiche Investitionen in den technischen Umweltschutz tätigen. Diese Maßnahmen werden im Rahmen verschiedener EU-Programme mit mehreren Milliarden Euro jährlich gefördert.

\section{Umwelteinstellungen der Bürger in den Mitglieds- und Beitrittsländern der eu- ropäischen Union}

In welchem Ausmaß unterstützen die Bürgerinnen und Bürger der verschiedenen europäischen Gesellschaften die Idee der EU, ein hohes Maß an Umweltschutz auch unter Beschränkung rein ökonomischer Rationalitätskriterien zu erzielen? Zur Beantwortung dieser Frage bedienen wir uns des Instrumentariums der international vergleichenden Surveyforschung. Wir beschreiben zunächst den verwendeten Datensatz und unsere methodische Vorgehensweise, stellen dann die deskriptiven Befunde vor und interpretieren die vorgefundenen Länderunterschiede.

\subsection{Daten, Variable und Methode}

Um die Umweltwerte der EU-Bürger zu analysieren, kann man auf verschiedene Datensätze zurückgreifen. Wir haben uns für den „Gallup International Millennium 
Survey“ aus dem Jahr 2000 entschieden. Der Millennium Survey ist eine standardisierte Einstellungsbefragung, die in 82 Ländern auf allen Kontinenten der Welt durch die „Gallup International Association“ (GIA), einem der weltweit größten kommerziellen Meinungsforschungsinstitute, durchgeführt wurde. ${ }^{8}$ Befragt wurden Personen ab 14 Jahren auf der Grundlage einer jeweils nationalen Zufallsauswahl. Die Befragungen fanden im Herbst 1999 statt und wurden mittels CATI-Interview oder, wo es aufgrund der nationalen technischen Voraussetzungen bzw. des Befragtenverhaltens nicht möglich war, als CAPI-Interview durchgeführt. Pro Land wurden unterschiedliche Stichprobengrößen realisiert (von 500 Befragten in den kleineren Ländern bis zu 2000 Befragten in den bevölkerungsreichen Ländern). Gegenstand der Befragung waren Einstellungen in folgenden Lebensbereichen: Umwelt, Governance und Demokratie, Religion, Gleichberechtigung der Geschlechter, Kriminalität, allgemeine Lebensziele, Vereinte Nationen und Menschenrechte.

Wir haben den Millennium Survey aus zwei Gründen ausgewählt. Erstens ist der Millennium Survey in der überwiegenden Mehrzahl der europäischen Staaten durchgeführt worden und deckt fast alle Mitglieds- und Beitrittsländer der EU ab. Von den EU-15-Ländern wurden lediglich Griechenland und Portugal nicht befragt, und von den 2004 beigetretenen Ländern (Beitritt-I) fehlen lediglich Slowenien und Malta. Die Beitrittsländer Rumänien, Bulgarien und die Türkei waren ebenfalls Teil der Befragung. Gegenüber anderen Umfragen wie etwa dem Eurobarometer 62.1, in dem ebenfalls eine Reihe von Umweltfragen gestellt wurden, sind im Millennium Survey damit auch die gegenwärtigen Beitrittskandidaten der EU komplett vertreten. Damit verfügen wir über einen Datensatz, der die Werte der überwiegenden Mehrheit der gegenwärtigen und zukünftigen EU-Bürgerinnen und Bürger abbildet. Zweitens enthält der Millennium Survey eine Frage, die das Umweltskript der EU vergleichsweise gut operationalisiert. Gefragt wurde, welches der beiden Ziele aus Sicht des Befragten wichtiger ist: die natürliche Umwelt zu schützen oder wirtschaftliches Wachstum sicherzustellen. "Which of the following statements do you tend to agree with more? It is more important to protect the environment than to ensure economic growth (1); It is more important to ensure economic growth than to protect the environment (2); Don't know (3)“. Zwischen den formulierten Zielen sollten die Befragten eine Entscheidung treffen.

Diese Frageformulierung hat einen doppelten Vorteil. Zum einen wird die Kostenseite des Umweltschutzes mit thematisiert. Es hat sich bei der Messung von Werteinstellungen herausgestellt, dass man diese valide operationalisieren kann, wenn man die Einnahme eines Wertestandpunktes mit bestimmten Konsequenzen („Kosten“) für das Individuum oder eine soziale Einheit verknüpft (vgl. zur so genannten „Low-cost Hypothese“ Diekmann 1996; Diekmann/Preisendörfer 2003). In unserem Fall lautet die Unterstellung in der Frageformulierung, dass die Förderung der Um-

8 Der Datensatz ist für wissenschaftliche Zwecke freigegeben und über die „Gallup International Association" bestellbar. 
welt mit materiellen Kosten verbunden ist: Wirtschaftliches Wachstum wird durch die Geltung von umweltpolitischen Normen begrenzt, weil die Einhaltung dieser Normen Kosten verursacht, für die der Einzelne und die Gemeinschaft aufkommen muss. Zum anderen misst die Frage recht gut das EU-Skript der EU. Wir hatten im letzten Kapitel gesehen, dass die Ökologievorstellungen Schritt für Schritt den Ökonomie- und Wachstumsvorstellungen zur Seite gestellt wurden. Die Frageformulierung enthält genau diese beiden Zielvorstellungen.

Wir geben die Antworten der Befragten pro Land in Form von relativen Häufigkeiten jeweils getrennt für den Vorrang des Umweltschutzes und des Wirtschaftswachstums wieder. Zusätzlich haben wir die Länder nach ihrem Beitrittszeitpunkt zur EU gruppiert.

Tabelle 1 gibt die Ergebnisse unserer Berechnung wieder. In 20 von 22 Ländern (Ausnahmen sind Ungarn und die Türkei) räumen die Bürger dem Umweltschutz eine höhere Priorität ein als wirtschaftlichem Wachstum. Dies sind auch im Vergleich $\mathrm{zu}$ anderen Wertsphären sehr hohe Zustimmungsraten (vgl. Gerhards/Hölscher 2005). Die Ökologievorstellungen der EU finden bei den Bürgern also eine, wie wir finden, erstaunliche hohe Unterstützung. Zwischen den Ländern und den vier Ländergruppen gibt es aber zum Teil deutliche Niveauunterschiede. Knapp 60 Prozent der EU-15-Bürger geben dem Umweltschutz den Vorzug vor wirtschaftlichem Wachstum. Nur in Deutschland sind es im Mittel weniger als 50 Prozent. Zwar erlaubt der Millenium-Datensatz keine Unterscheidung der Befragten in Ost- und Westdeutschland. Wie wir jedoch im nächsten Abschnitt sehen werden, dürfte der in Relation zum EU-15-Durchschnitt niedrigere Mittelwert auf geringere Zustimmungen in Ostdeutschland zurückgehen. In den Beitritt I- und Beitritt II-Ländern sind es noch rund 48 Prozent und in der Türkei nur noch 40,5 Prozent der Befragten, die für eine herausgehobene Rolle des Umweltschutzes plädieren. 
Tabelle 1: Umweltwerte in der Europäischen Union und den Beitrittskandidaten

\begin{tabular}{|c|c|c|c|}
\hline & $\begin{array}{l}\text { Umweltschutz ist wich- } \\
\text { tiger als ökonomisches } \\
\text { Wachstum (in \%) }\end{array}$ & $\begin{array}{l}\text { Ökonomisches Wachs- } \\
\text { tum ist wichtiger als } \\
\text { Umweltschutz (in \%) }\end{array}$ & $\begin{array}{c}\text { Weiß nicht } \\
\text { (in } \%)\end{array}$ \\
\hline EU-15 & 59,7 & 25,4 & 15,0 \\
\hline Dänemark & 72,5 & 15,2 & 12,3 \\
\hline Finnland & 69,8 & 15,3 & 14,9 \\
\hline Großbritannien & 65,5 & 25,4 & 9,1 \\
\hline Schweden & 62,2 & 29,4 & 8,4 \\
\hline Spanien & 62,0 & 17,9 & 20,1 \\
\hline Luxemburg & 60,4 & 26,8 & 12,8 \\
\hline Niederlande & 59,8 & 19,3 & 21,0 \\
\hline Italien & 59,5 & 26,0 & 14,5 \\
\hline Irland & 57,3 & 23,2 & 19,5 \\
\hline Belgien & 56,1 & 33,9 & 10,0 \\
\hline Österreich & 53,3 & 21,0 & 25,5 \\
\hline Frankreich & 52,7 & 43,2 & 4,1 \\
\hline Deutschland & 44,3 & 33,4 & 22,3 \\
\hline Beitritt I & 48,2 & 40,3 & 11,5 \\
\hline Slowakei & 57,2 & 39,9 & 2,9 \\
\hline Litauen & 49,0 & 45,9 & 5,2 \\
\hline Estland & 48,9 & 38,2 & 12,9 \\
\hline Polen & 47,4 & 35,8 & 16,7 \\
\hline Lettland & 45,8 & 37,1 & 17,1 \\
\hline Ungarn & 41,0 & 44,9 & 14,1 \\
\hline Tschechien* & - & - & - \\
\hline Beitritt II & 47,9 & 21,8 & 30,3 \\
\hline Rumänien & 47,9 & 21,9 & 30,2 \\
\hline Bulgarien & 47,8 & 21,8 & 30,3 \\
\hline Türkei & 40,5 & 44,4 & 15,1 \\
\hline
\end{tabular}

Blicken wir auf die zweite Spalte, so finden wir eine annähernd spiegelbildliche Struktur vor. Während nur rund ein Viertel der Befragten EU-15-Bürger für den Vorrang der Wirtschaft plädiert, sind es in den Beitritt-I-Ländern und der Türkei mehr als 40 Prozent. In den Beitritt-II-Ländern ist dagegen ein großer Teil der Befragten ambivalent. Während sich nur rund 22 Prozent der Bürger für den Vorrang des ökonomischen Wachstums aussprechen, sehen sich 30 Prozent nicht in der Lage, zwischen beiden Wertorientierungen eine Entscheidung zu treffen.

Aber auch innerhalb der Ländergruppen finden sich zum Teil deutliche Varianzen. Die höchsten Zustimmungsraten erhält der Umweltschutz in Skandinavien, ge- 
folgt von den westeuropäischen Ländern Großbritannien, den Benelux-Staaten, Italien und Spanien sowie der Slowakei. Ähnlich wie in anderen Wertebereichen bilden die skandinavischen Länder die „Musterknaben“ der EU (vgl. Gerhards/Hölscher 2005). Die niedrigste Zustimmung findet der Umweltschutz in Lettland, Ungarn sowie der Türkei. Diese Ergebnisse decken sich weitgehend mit Berechnungen auf der Grundlage anderer Datensätze wie dem ISSP 2000 (Franzen/Meyer 2000: 124), dem Eurobarometer 62.1 (European Commission 2005: 41 ff.) und dem World Values Survey 1990-93 (Inglehart 1995: 61).

Fazit: Insgesamt sind die Zustimmungsraten der Bürgerinnen und Bürger zum Ökologieskript der EU recht hoch; Umweltschutz ist ein fester Bestandteil der Werte der Bürger Europas. Im Vergleich zu anderen Wertsphären befinden sich die Wertvorstellungen der Bürger im Einklang mit den Wertvorstellungen der Institutionen der EU. Dies gilt aber nicht für alle Länder gleichermaßen. Mit der vollzogenen Aufnahme der mittelosteuropäischen Beitrittsländer sowie mit der nächsten Erweiterung im Jahr 2007 wird sich das kulturelle Gesamtgefüge der EU im Hinblick auf die Ökologievorstellungen aber verändern: Der Anteil derer, die die Wirtschaftspolitik der EU durch ökologische Rationalitäten ergänzt sehen wollen, wird abnehmen. Dieses Ergebnis wird mittelfristig Folgen für das Institutionengefüge der EU und für die politischen Akteure zeitigen. Vor allem für die Akteure, die sich das Ökologiethema auf die Fahnen geschrieben haben, werden sich mit der weiteren Expansion der EU die Bedingungen für die Durchsetzung ihrer Ziele verschlechtern. Die Erweiterung wird den Gegenwind für diese Interessengruppen erhöhen, weil der Rückhalt für ihre Forderungen, die sie in der Vergangenheit vor allem auf der Ebene der EUKommission relativ erfolgreich durchsetzen konnten (vgl. Roose 2003), schwächer wird. Mit der Mitgliedschaft der neuen Länder erhalten diese auch alle Mitspracheund Gestaltungsrechte in den EU-Gremien. Damit verschieben sich die Machtverhältnisse innerhalb der Institutionen der EU. Ökologiefragen werden es daher möglicherweise schwerer haben, sich politisch Gehör zu verschaffen. Die Repräsentanten der neuen Länder werden für diese Fragen weniger Interesse aufbringen, wenn sie sich an den Vorstellungen ihrer Bürger orientieren.

Von den mittelfristigen Folgen sind die langfristigen Effekte zu unterscheiden. Werthaltungen sind keine unveränderlichen Größen, sie können sich in Abhängigkeit von den sozialen Kontextbedingungen verändern (vgl. statt anderer Inglehart 1989, 1997; Kohn/Schooler 1982; Wegener 2001). Würden sich in den Beitrittsländern die Kontextbedingungen der Übernahme von Umweltwerten ändern, so hätte dies langfristig eine Zunahme an Unterstützung für die umweltpolitischen Zielvorstellungen der EU zur Folge. Damit stellt sich die Frage, welche sozialen Kontexte Umwelteinstellungen beeinflussen. 


\section{Erklärung der Unterschiede in den Umwelteinstellungen}

\subsection{Hypothesen}

Wir gehen davon aus, dass Einstellungen zum Umweltschutz von drei Bedingungen abhängen: (1) von der materiellen Wohlfahrt der Bürger und ihrer Länder, (2) von den grundlegenden Werthaltungen der Bürger, und (3) von der Umweltqualität eines Landes.

(1) Umweltschutz als Budgetrestriktion. Wir vermuten, dass die Bürger Umweltschutz insofern als eine Budgetrestriktion wahrnehmen, als dass sie Maßnahmen des Umweltschutzes vorwiegend mit Kostenfaktoren assoziieren und weniger als Quelle des wirtschaftlichen Fortschritts ansehen (vgl. statt anderer Diekmann 1996). Und in der Tat sind ja eine Reihe von Umweltschutzmaßnahmen - wie der Einbau von Filteranlagen oder die Einrichtung von Landschaftsschutzgebieten - mit erheblichen Kosten verbunden. Diese Kosten sind vom Einzelnen und der Gemeinschaft aufzubringen. Damit konkurriert der Umweltschutz nicht nur mit den materiellen Nutzeninteressen der Bürger, sondern auch mit einer Reihe anderer Werte, deren Umsetzung in die Praxis ebenfalls materielle Kosten hervorruft (z.B. die Herstellung eines soziale Gerechtigkeit befördernden Sozialstaats oder die Gleichberechtigung der Geschlechter auf dem Arbeitsmarkt). Daher vermuten wir, dass Präferenzen für den Schutz der Umwelt zum einen vom Niveau des individuellen Wohlstands abhängen: Je wohlhabender ein Bürger ist, desto geringer ist seine Budgetrestriktion für Investitionen in den Umweltschutz, und desto mehr spricht er sich für Umweltschutz als Ziel aus (vgl. auch Franzen/Meyer 2004).

Diese These deckt sich mit der sog. „Luxusgutthese“ in der Ökonomik, wonach die Bereitschaft zum Umweltengagement mit steigendem individuellem Wohlstand zunimmt (vgl. Baumol/Oates 1979: 174 ff.). Allerdings ist die Luxusgutthese durchaus umstritten. So hat beispielsweise Peter Preisendörfer anhand einer deutschen Bevölkerungsumfrage keine signifikanten Einstellungsunterschiede zwischen verschiedenen Einkommensgruppen gefunden (Preisendörfer 1999: 130 ff.). Wir messen den individuellen Wohlstand daher nicht allein durch die Höhe des (logarithmierten) Familieneinkommens, das einer Person zur Verfügung steht, sondern zugleich durch seinen sozialen Status (Klassenlage). Indem wir den sozialen Status einbeziehen, folgen wir der in der Literatur geäußerten Ansicht, dass die Einkommenslage allein keinen zureichenden Prädiktor für Einstellungen zum Umweltschutz darstellt (vgl. Preisendörfer/Franzen 1996: 232).

H1a: Je höher das Familieneinkommen einer Person bzw. je höher ihr sozialer Status, desto stärker ist ihre Präferenz für Umweltschutz.

Der gleiche Zusammenhang gilt für die Budgetrestriktion eines Landes, in dem ein EU-Bürger wohnt. Da eine intakte Umwelt ein kollektives Gut ist, für dessen Erhal- 
tung bzw. Wiederherstellung geringe individuelle Anreize bestehen, müssen viele Umweltschutzmaßnahmen öffentlich finanziert werden. Je höher nun diese Ausgaben sind, desto größer ist die Budgetrestriktion des Staates für die Durchsetzung konkurrierender Werte. In Übereinstimmung mit anderen Studien (Diekmann/Franzen 1999; Franzen 2003; Preisendörfer/Franzen 1996: 228 ff.) vermuten wir, dass die Zustimmung der Bürger zum Umweltschutz von der ökonomischen Wohlfahrt ihres Landes abhängt: Je reicher das Land, desto stärker die Präferenz für den Umweltschutz. Misst man die materielle Wohlfahrt eines Landes über das Bruttoinlandsprodukt pro Einwohner, so lautet unsere nächste Hypothese:

H1b: Je höher das Bruttoinlandsprodukt eines Landes, desto stärker sind die Präferenzen seiner Bürger für den Umweltschutz.

(2) Grundlegende Wertvorstellungen und Umwelteinstellungen. Menschen folgen nicht nur ihren materiellen Interessen, sondern auch ihren Ideen und ideologischen Orientierungen (vgl. den Überblick über experimentelle Ergebnisse bei Fehr/Fischbacher 2002). Das Links/Rechts-Schema stellt ein abstraktes ideologisches Raster dar, mit dem die Bürger konkrete politische Themen interpretieren. Es erleichtert den Bürgern eine Orientierung und eine eigene Positionsbestimmung bezüglich einer Vielzahl unterschiedlicher konkreter politischer Themen. Mit den Polen rechts und links sind jeweils unterschiedliche generelle gesellschaftliche Werte und politische Ideologien verbunden. Dieter Fuchs und Hans-Dieter Klingemann (1990) haben diese Vorstellungen auf der Basis einer Umfrage in drei Ländern empirisch rekonstruiert. Mit rechten Vorstellungen sind im stärkeren Maße Ideen der wirtschaftlichen Entwicklung, des Wachstums und der nationalen Identität verbunden, mit linken Vorstellungen im höheren Maße Ideen der Gleichheit und Solidarität. Wie verschiedene empirische Studien zeigen, gilt dies offenbar auch für Einstellungen zum Umweltschutz (vgl. Preisendörfer 1999: 156; Preisendörfer/Franzen 1996: 228). Im Anschluss an diese Befunde vermuten wir, dass sich Personen, die sich als politisch links einschätzen, verstärkt für Umweltschutz aussprechen, während Bürger mit rechtsgerichteten Überzeugungen eher gegen Umweltschutz votieren:

H2a: Personen mit linker politischer Orientierung sprechen sich stärker für Umweltschutz aus als Personen mit rechter politischer Orientierung.

In Hypothese $1 \mathrm{~b}$ haben wir einen Effekt des Wohlstandsniveaus eines Landes auf die Umweltwerte seiner Bevölkerung formuliert. Wir greifen diesen Zusammenhang nochmals auf, diesmal jedoch unter anderen theoretischen Vorzeichen. Wie die Postmaterialismus-These von Ronald Inglehart behauptet, geht mit der Steigerung des ökonomischen Wohlstands eine Veränderung der grundlegenden Werthaltungen der Bürger einher (Inglehart 1971; 1983; 1989; 1997). Die Zunahme der Möglichkeit der Befriedigung materieller Bedürfnisse führt mit einer Zeitverzögerung, so die 
Inglehartsche These, zu einer Werteverschiebung von materialistischen hin zu postmaterialistischen Werten. Materielle Werte sind solche der Befriedigung der ökonomischen Lebensbedingungen und der Sicherheit, und zu den postmateriellen Werten gehören vor allem Wünsche nach Selbstentfaltung und Partizipation. Folgt man Inglehart, so werden sich Menschen mit postmaterialistischen Einstellungen verstärkt für Umweltschutz aussprechen, da die intakte Umwelt als Voraussetzung der Selbstentfaltung interpretiert wird (Inglehart 1995; s. a. Betz 1990). Wir erwarten daher, dass sich Personen mit postmaterialistischen Wertvorstellungen stärker für Umweltschutz aussprechen werden als Personen mit materialistischen Werten. Entscheidend ist, dass dieser Effekt unabhängig von der aktuellen Budgetrestriktion einer Person oder der ihres Landes zustande kommt. Wie man aus der Sozialisationsforschung weiß, eignen sich Menschen ihre grundlegenden Werte in der Adoleszenz an (vgl. Inglehart 1983; Nunner-Winkler 2000). Diese Werthaltungen bleiben auch dann relativ stabil, wenn sich die ökonomische Lage eines Individuums oder seines Landes zu späteren Zeitpunkten ändert (vgl. Franzen/Meyer 2004: 121).

H2b: Personen mit postmaterialistischen Wertvorstellungen sprechen sich stärker für Umweltschutz aus als Personen mit materialistischen Wertvorstellungen.

Wie verschiedene Studien gezeigt haben, nimmt das Umweltbewusstsein mit steigendem Alter ab (Diekmann/Franzen 1999; Greenbaum 1995; Franzen/Meyer 2004; Langhenne/Lehmann 1986; Preisendörfer/Franzen 1996). Verantwortlich dafür ist ein Kohorteneffekt. Die Wertesozialisation der nach 1960 in den alten EU-Ländern geborenen Kohorten fand zu einer Zeit statt, in der Umweltschäden wie Luft- und Wasserverschmutzung als auch die Risiken der Kernkraftnutzung in zunehmendem Maße in der Öffentlichkeit als Problem der gesellschaftlichen Entwicklung wahrgenommen wurden. Dagegen waren Umweltprobleme zur Zeit der Wertesozialisation der vor 1960 Geborenen kaum ein Thema der öffentlichen Debatte. Wir vermuten daher, dass das Alter als Proxy für die Geburtskohorte eines EU-Bürgers einen von postmaterialistischen Überzeugungen eigenständigen Effekt auf die Bereitschaft zum Engagement für die Umwelt aufweist:

H2c: Je jünger eine Person ist, desto stärker spricht sie sich für Umweltschutz aus.

(3) Umweltqualität. Unsere dritte Hypothese thematisiert den Zusammenhang zwischen dem objektiven Zustand der natürlichen Umwelt und dem Umweltbewusstsein. Manche Autoren gehen davon aus, dass ein höheres Umweltbewusstsein die Folge eines schlechten lokalen bzw. nationalen Umweltzustandes ist. So zeigt Inglehart (1995) anhand der Daten des „World Values Survey“ (1990-93), dass mit höherer Luftverschmutzung in einem Land die Zustimmung seiner Bürger zum Umweltschutz ansteigt. Trotz einer anderen theoretischen Ausgangsposition gelangt auch Riley Dunlap zu ähnlichen Ergebnissen (Dunlap 1995; Dunlap et al. 1993; Dun- 
lap/Mertig 1996). Auf der Basis des Gallup „Health of the Planet Survey“ von 1993 kommt er zu dem Schluss, dass je schlechter es in einem lokalen Raum, einer Region oder einem Land um die Umwelt bestellt ist, desto betroffener zeigen sich die Bürger für Umweltprobleme, und desto größer ist der Stellenwert des Umweltschutzes im Rahmen des individuellen Wertekatalogs. Unsere dritte Hypothese lautet entsprechend:

H3: Je schlechter die Umweltqualität eines Landes ist, desto stärker sind die individuellen Präferenzen für Umweltschutz.

\subsection{Daten, Methode und Variablen}

Wir können für die Kausalanalyse nicht auf die Daten des Gallup Millennium Survey zurückgreifen, da in diesem Datensatz keine geeigneten Informationen über die sozioökonomische Lage der Befragten und über grundlegende politische Werthaltungen erhoben wurden, weshalb Informationen über entscheidende unabhängige Variablen fehlen. Wir verwenden stattdessen die Daten des „International Social Science Programme (ISSP)“ aus dem Jahr 2000 ( „Environment II“). Das ISSP ist ein seit 1983 existierendes internationales Programm zur Koordination ländervergleichender Umfragen mit jeweiligen Themenschwerpunkten. Im Jahr 2000 wurden weltweit in 26 Ländern Einstellungen zur Umwelt erhoben. Die nationalen Zufallsstichproben umfassen zwischen 900 und 1500 Befragte. Zum Einsatz kamen - je nach Land - standardisierte schriftliche Fragebogen (Selbstausfüller) oder Face-to-face-Interviews.

Der ISSP-Datensatz „Environment II“ hat allerdings den Nachteil, dass er nicht die Breite der EU-Länder abdeckt. Im Rahmen des ISSP wurden Antworten von Personen aus zehn EU-15-Ländern (Ost- und Westdeutschland wurden getrennt erhoben und können auch getrennt analysiert werden), aus nur drei Beitritt-I Ländern sowie nur einem Beitritt-II Land erhoben. ${ }^{9}$ Die Anzahl der europäischen Länder ist damit sehr begrenzt, was die Validität unserer Analysen durchaus beeinträchtigen kann. Wir haben uns dennoch aus mehreren Gründen für die Analyse dieses Datensatzes entschieden. Erstens enthält der Datensatz die im letzten Abschnitt beschriebenen unabhängigen Variablen, zudem eine Vielzahl an Einstellungsfragen zum Umweltschutz.

Zweitens stellt der Datensatz, anders als z. B. die neuere EurobarometerBefragung 62.1, Daten aus jedem unserer drei EU-Ländergruppen bereit. Drittens gehen wir davon aus, dass die von uns postulierten Wirkungszusammenhänge prinzipiell in allen Ländern gelten, d. h. die Länder stellen jeweils nur Spezifikationen der jeweiligen Randbedingungen (z.B. des Grads der ökonomischen Modernisie-

9 Da nicht alle von uns verwendeten Variablen in allen Ländern erhoben wurden, reduziert sich für unsere Analysen die Zahl der EU-15 Länder auf neun (inklusive Ostdeutschland). 
rung) dar. Diese Annahme können wir jedoch nur unter der Voraussetzung treffen, dass die Struktur der Umweltwerte in den ISSP-Ländern in etwa der Struktur der Zustimmung in den Gallup-Ländern entspricht. Ob dies der Fall ist, können und werden wir überprüfen.

Zur Messung von Umweltwerten in Konkurrenz zu Wirtschaftswerten stehen uns verschiedene Items zur Verfügung, die jeweils das Verhältnis von Wirtschaft und Umwelt auf der individuellen Ebene abbilden. Eine Frage stellt den Befragten vor die Aufgabe, auf einer fünfstufigen Zustimmungsskala anzugeben, in welchem Ausmaß er bereit wäre, seinen Lebensstandard zum Zwecke der Förderung der Umwelt abzusenken: "And how willing would you be to accept cuts in your standard of living in order to protect the environment?" Wir geben diesem Item den Vorzug vor indexbasierten Umweltbewusstseinsmessungen, weil es am ehesten unserer Messstrategie aus Abschnitt 3 entspricht, nämlich die Einnahme eines Umweltstandpunktes mit der Konsequenz materieller Kosten zu verknüpfen. Wir haben aber zusätzlich zu den im Folgenden dargestellten Regressionsanalysen auch mit einem Index (gebildet aus vier Items) eine Kausalanalyse durchgeführt. Die Befunde beider Analysen sind weitgehend deckungsgleich (vgl. dazu Anhang 3).

Zur Prüfung unserer Hypothesen haben wir lineare hierarchische Regressionsanalysen durchgeführt. Die Beschreibung der Variablen zur Operationalisierung der theoretischen Konstrukte befindet sich in Anhang 2. Das Basismodell überprüft den Einfluss des Alters und des materiellen Wohlstands einer Person auf die Einstellung zum Umweltschutz. Modell zwei enthält zusätzlich die Effekte der grundlegenden Werthaltungen. Im dritten Modell fügen wir das Bruttoinlandsprodukt pro Einwohner (in Kaufkraftparitäten) und den ESI-Index, der Umweltqualität in einem Land misst, als Aggregatsmerkmale der Länder hinzu. Da pro Land mehrere Personen befragt werden, müssen wir davon ausgehen, dass ein unbeobachteter Einfluss der Länderzugehörigkeit auf die Zustimmung der Befragten zum Umweltschutz besteht. In diesem Fall sind die Modellannahmen der Regressionsanalyse verletzt. Aus diesem Grund schätzen wir robuste Standardfehler unter Berücksichtigung von Landesclustern (Huber-Regression; vgl. Huber 1967). ${ }^{10}$

10 Die Modellierung eines hierarchischen Regressionsmodells scheint auf den ersten Blick ungerechtfertigt, da die Modellannahmen der Regression aufgrund der Zwei-Ebenen-Struktur der Daten (Individual- und Kontextvariablen) grundsätzlich verletzt zu sein scheinen. Sollten Cross-LevelEffekte zwischen den verwendeten Variablen vorliegen, so dass der Effekt der individuumsbezogenen Variablen auf die Umweltwerte von der Ausprägung der Kontextvariablen abhängig ist, müsste in der Tat ein Mehrebenenmodell modelliert werden. Aufgrund unserer theoretischen Annahmen gehen wir jedoch für keine der beiden Kontextvariablen „Bruttoinlandprodukt“ und „Umweltqualität“ davon aus, dass sie den Einfluss der individuellen Merkmale auf die ökonomische Verzichtsbereitschaft zugunsten des Umweltschutzes moderiert. Daher beschränken wir uns auf eine Huber-Regression. 


\subsection{Ergebnisse}

In welchem Maße zeigen sich in den ISSP-Daten ähnliche Länderunterschiede wie in den Daten des Gallup Millenium Survey? Tabelle 2 gibt darüber Auskunft. Die Verteilung der Länder deckt sich weitgehend mit der aus Tabelle 1. Wiederum äußern die Bürger in den EU-15 Ländern die umweltfreundlichsten Einstellungen, wobei Österreich und Westdeutschland sowie die skandinavischen Länder die vorderen Plätze einnehmen. Es folgen die neuen Beitritt-I-Länder sowie das Beitritt-II-Land Bulgarien. Lettland liegt mit nur 6 Prozent Zustimmung und 78 Prozent Ablehnung auf dem letzten Platz.

Tabelle 2: Lebensstandard absenken für Umweltschutz (relative Häufigkeiten)

\begin{tabular}{lccc}
\hline & Zustimmung & Ablehnung & weder / noch \\
EU-15 & $\mathbf{3 5 , 0}$ & $\mathbf{3 9 , 8}$ & $\mathbf{2 5 , 2}$ \\
Österreich & 50,6 & 28,7 & 20,8 \\
Schweden & 44,9 & 27,3 & 27,8 \\
Deutschland (West) & 43,3 & 27,4 & 29,3 \\
Finnland & 42,3 & 30,2 & 27,5 \\
Irland & 35,3 & 48,0 & 16,7 \\
Dänemark & 32,8 & 34,0 & 33,2 \\
Spanien & 30,9 & 44,8 & 24,3 \\
Deutschland (Ost) & 26,6 & 47,0 & 26,4 \\
Großbritannien & 26,3 & 49,9 & 23,8 \\
Portugal & 16,8 & 60,8 & 22,4 \\
\hline Beitritt I & $\mathbf{2 0 , 2}$ & $\mathbf{5 6 , 3}$ & $\mathbf{2 3 , 5}$ \\
Slowenien & 33,8 & 30,3 & 35,9 \\
Tschechien & 21,0 & 60,6 & 18,4 \\
Lettland & 5,8 & 78,1 & 16,1 \\
\hline Beitritt II & $\mathbf{1 2 , 1}$ & $\mathbf{6 9 , 1}$ & $\mathbf{1 8 , 8}$ \\
Bulgarien & & & \multirow{2}{*}{ Quer } \\
\hline Quelle: ISSP 2000, N = 15467. Operationalisierung: Zusammenfassung der beiden Zu- & &
\end{tabular}

Die Ergebnisse unserer multivariaten Berechnungen sind in Tabelle 3 festgehalten. Blicken wir zunächst auf unser Ausgangsmodell in der zweiten Spalte. Je jünger ein Befragter ist, desto stärker ist er bereit, seinen Lebensstandard für den Umweltschutz abzusenken. Andere Studien kommen zu ähnlichen Ergebnissen (Diekmann/Franzen 1999; Greenbaum 1995; Franzen/Meyer 2004). Hypothese H2c wird also bestätigt, die Kohorten unterscheiden sich bezüglich ihrer Umwelteinstellungen. 
Tabelle 3: Kausalanalyse „Lebensstandard absenken für Umweltschutz"

\begin{tabular}{|c|c|c|c|}
\hline & Modell 1 & Modell 2 & Modell 3 \\
\hline \multicolumn{4}{|l|}{ Soziodemografische Merkmale } \\
\hline \multirow{2}{*}{ Alter (in Jahren) } &,$- 045^{*}$ &,$- 036^{*}$ &,$- 043^{*}$ \\
\hline & $(-2,67)$ & $(-2,41)$ & $(-2,93)$ \\
\hline \multirow{2}{*}{ Familieneinkommen } & ,103 & ,098 & 050 \\
\hline & $(1,59)$ & $(1,59)$ & $(0,92)$ \\
\hline Klassenlage & $138^{* * *}$ & $132^{* * *}$ &, $098^{* * *}$ \\
\hline (Selbsteinstufung) & $(4,37)$ & $(4,78)$ & $(4,66)$ \\
\hline \multicolumn{4}{|l|}{ Generalisierte Einstellungen } \\
\hline \multirow{2}{*}{$\begin{array}{l}\text { Politische Selbsteinstufung } \\
\text { (Links-Rechts-Skala) }\end{array}$} & &,$- 068^{*}$ &,- 054 \\
\hline & & $(-2,26)$ & $(-1,96)$ \\
\hline \multicolumn{2}{|l|}{ Postmaterialismus } & $135^{* * *}$ &, $110^{* * *}$ \\
\hline \multicolumn{2}{|l|}{ (Inglehart-Index) } & $(8,55)$ & $(8,71)$ \\
\hline \multicolumn{4}{|l|}{ Ländermerkmale } \\
\hline \multirow{2}{*}{ GDP per capita (PPP) } & & &, $177^{*}$ \\
\hline & & & $(2,94)$ \\
\hline \multirow{2}{*}{ Umweltqualität (ESI-Index) } & & & ,009 \\
\hline & & & $(0,19)$ \\
\hline $\mathbf{R}^{2}$ & , 037 & ,061 & ,090 \\
\hline
\end{tabular}

Wir erwarten weiterhin (Hypothese H1a), dass sich die Höhe des Einkommens und der Sozialstatus positiv auf die Präferenzen für Umweltschutz auswirken. Die Ergebnisse bestätigen diese Hypothese nur teilweise. Einerseits finden wir einen hochsignifikanten Effekt der subjektiven Klassenlage eines Befragten auf die Umwelteinstellungen, der über alle drei Modelle stabil bleibt. Und auch das Familieneinkommen wirkt in die vermutete Richtung. Andererseits bleibt der letztgenannte Effekt insignifikant. Dies ändert sich auch dann nicht, wenn man die beiden Sozialstrukturvariablen einzeln in das Modell einfügt. Da die empirische Forschungslage, wie erläutert, bezüglich des Einflusses des Einkommens auf Umwelteinstellungen widersprüchlich ist (vgl. Preisendörfer 1999; Franzen/Meyer 1999), vermuten wir, dass das Familieneinkommen eine zu enge Operationalisierung des individuellen Wohlstands einer Person darstellt.

In Modell 2 sind die beiden generalisierten Einstellungsvariablen hinzugefügt. Die Koeffizienten der Variablen in Modell 1 werden dadurch nicht wesentlich verändert, was für die Stabilität unserer Modelle spricht. Es zeigt sich, dass sich die Befragten (im Sinne unserer Annahme H2a) dann verstärkt für Umweltschutz aussprechen, wenn sie sich selbst als politisch links einschätzen. Allerdings ist dieser Effekt nur schwach signifikant (im dritten Modell nur auf dem 7 Prozent-Niveau). Für unsere Hypothese $\mathrm{H} 2 \mathrm{~b}$ finden wir dagegen klare empirische Evidenz: Personen mit postma- 
terialistischen Einstellungen berichten eine höhere Sensibilität für die Belange der Umwelt als Personen mit materialistischen Werthaltungen.

Modell 3 enthält zusätzlich die beiden Makrovariablen. Wie in Hypothese H1b postuliert, nimmt mit steigendem Bruttoinlandsprodukt pro Kopf die Zustimmung zum Umweltschutz zu. Entgegen der Hypothese H3 finden wir keinen negativen Zusammenhang zwischen der Umweltqualität eines Landes und den Umweltwerten seiner Bürger. Aufgrund des niedrigen t-Werts lässt sich das positive Vorzeichen des Koeffizienten nicht inhaltlich interpretieren.

Vergleichen wir abschließend die standardisierten Effektstärken der unabhängigen Variablen im Endmodell 3, so sehen wir, dass das Ländermerkmal des Bruttoinlandsprodukts pro Kopf den vergleichsweise stärksten Effekt hervorruft, gefolgt vom Postmaterialismus-Index und der Klassenlage einer Person. Die aufgeklärte Varianz ist mit knapp 10 Prozent zwar nicht allzu groß. Allerdings muss man bedenken, dass es uns nicht darum ging, eine möglichst umfangreiche Erklärung von Umwelteinstellungen vorzulegen, sondern lediglich darum, einige spezielle, für die langfristige Entwicklung der EU-Umweltpolitik maßgebliche Hypothesen zu überprüfen. Deswegen haben wir auch darauf verzichtet, zahlreiche andere Einflussgrößen (Geschlecht, Bildung, Beruf, Erwerbsstatus, Kinderzahl, Umweltengagement etc.) in die Modelle zu integrieren. ${ }^{11}$

Welche Schlussfolgerungen lassen sich nun aus unseren Kausalanalysen für die zukünftige Unterstützung des Ökologieskripts der EU durch die Bürgerinnen und Bürger der neuen Mitgliedstaaten ziehen? Die Präferenz für Umweltschutz hängt neben der Kohortenzugehörigkeit einer Person und ihrer Klassenlage vor allem vom Grad der ökonomischen Modernisierung der Länder und den grundlegenden Wertorientierungen der Bürger ab, wobei die postmaterialistischen Einstellungen wiederum vom Grad der Modernisierung eines Landes abhängen. Die Beitritt-I und BeitrittII-Länder sowie die Türkei liegen in diesen beiden Dimensionen deutlich hinter den alten Mitgliedstaaten zurück. Doch dies kann sich langfristig ändern. Gelingt eine ökonomische Modernisierung der neuen Mitgliedstaaten ähnlich gut wie in der Vergangenheit im Falle von Spanien, Griechenland oder Irland (vgl. Delhey 2003; Bornschier et al. 2004), so ist langfristig mit einer stärkeren normativen Unterstützung des Umweltschutzes zu rechnen.

11 Zur Absicherung unserer Befunde haben wir zusätzlich eine Regression mit dem MilleniumDatensatz gerechnet. Darin fungierte die Frage nach dem Vorrang des Umweltschutzes vor Wirschaftswachstum als (dichotome) abhängige Variable sowie Postmaterialismus, Bruttoinlandsprodukt und Umweltqualität als unabhängige Variablen. Die Ergebnisse bestätigen die Befunde unserer Analyse des ISSP-Datensatzes. 


\section{Bilanz}

In diesem Beitrag sind wir der Frage nachgegangen, in welchem Maße die Bürgerinnen und Bürger die institutionalisierten Ökologiewerte der EU unterstützen. In einem ersten Schritt haben wir unter Rückgriff auf die Entwicklung des Primär- und Sekundärrechts das Ökologieskript der EU rekonstruiert. Die EU-Umweltpolitik hat seit den 70er Jahren enorm an Bedeutung gewonnen, so dass die Idee einer zu schützenden Umwelt heute zum festen Bestandteil des Gesellschaftsskripts der EU gehört. Die EU erwartet von ihren Mitgliedstaaten den aktiven Einsatz für den Schutz der natürlichen Umwelt auch um den Preis der Beschränkung wirtschaftlicher Freiheiten und erheblicher finanzieller Aufwendungen.

$\mathrm{Ob}$ und in welchem Maße diese Werte von den Bürgerinnen und Bürgern der EUMitglieds- und Beitrittsländern geteilt werden, haben wir anhand der Analyse von Umfragedaten des "Gallup Millennium Survey“ geprüft. Insgesamt sind die Zustimmungsraten der Bürgerinnen und Bürger zu dem Ökologieskript der EU recht hoch; Umweltschutz ist ein fester Bestandteil der Werte der Bürger Europas. Dies gilt aber nicht für alle Länder gleichermaßen. Wir haben gesehen, dass sich die Bürger der EU-15-Länder stärker für den Vorrang der Umwelt vor der Wirtschaft aussprechen, als dies die Bürger der Beitritt I- und Beitritt II-Länder sowie der Türkei tun.

Was aus diesen Befunden für das Institutionengefüge der EU und für die weitere Entwicklung der EU-Umweltpolitik folgt, haben wir anhand der Unterscheidung in mittelfristige und langfristige Effekte darzulegen versucht. Mittelfristig kann man erwarten, dass die mittelosteuropäischen Länder über die in den Beitrittsverhandlungen festgelegten Maßnahmen zur Verbesserung der Umweltqualität kaum weitergehende EU-Initiativen zum Umweltschutz unterstützen werden. Die Repräsentanten der neuen Länder werden für diese Fragen weniger Interesse aufbringen, wenn sie sich an den Vorstellungen ihrer Bürger orientieren. Langfristig jedoch kann die Bereitschaft zum Umweltschutz jedoch ansteigen, nämlich dann, wenn (1) die erwartete ökonomische Modernisierung der neuen Mitgliedstaaten voranschreitet, und wenn sich (2) in ihrem Gefolge die grundlegenden Werthaltungen vom Materialismus hin zum Postmaterialismus ändern. $\mathrm{Zu}$ diesem Befund sind wir auf der Basis von multivariaten Analysen von ISSP-Daten gelangt. Denn in beiden Fällen würde der Umweltschutz in den neuen Mitgliedstaaten an Zustimmung gewinnen, was die Bereitschaft der jeweiligen Regierungen erleichtern würde, umweltpolitische Initiativen der Kommission oder des Parlaments zumindest nicht zu blockieren. 


\section{Literatur}

Almond, G. A. / Powell, G. B. / Strom, K. / Dalton, R. J., 2003: Comparative Politics Today. New York: Longman.

Bailey, I., 2003: New Environmental Policy Instruments in the European Union. Aldershot: Ashgate.

Barnes, P.M. / Barnes, I.G., 1999: Environmental Policy in the European Union. Cheltenham etc.: Elgar.

Betz, H.-G., 1990: Value Change in Postmaterialist Politics: The Case of West Germany. Comparative Political Studies 23: 239-256.

Bornschier, V. / Herkenrath, M. / Ziltener, P., 2004: Political and Economic Logic of Western European Integration. A Study of Convergence Comparing Member and Non-member States 1980-98. European Societies 6: 71-96.

Bundesministerium für Umwelt, Naturschutz und Reaktorsicherheit (BMU), 2005: Umweltschutz und Beitrittsvertrag, http://www.bmu.de/europa_und_umwelt/eu-erweiterung/doc/2244.php (Download am 13.07.2005).

Delhey, J., 2003: Europäische Integration, Modernisierung und Konvergenz. Zum Einfluss der EU auf die Konvergenz der Mitgliedsländer. Berliner Journal für Soziologie: 565-584.

Diekmann, A. / Franzen, A., 1999: The Wealth of Nations and Environmental Concern. Environment and Behavior 31: 540-549.

Diekmann, A. / Preisendörfer, P., 2003: Green and Greenback: The Behavioral Effects of Environmental Attitudes in Low-Cost and High-Cost Situations. Rationality and Society 15: 441-472.

Diekmann, A., 1996: Anwendungen und Probleme der Theorie rationalen Handelns im Umweltbereich. S. 89-118 in: A. Diekmann / C.C. Jaeger (Hrsg.): Umweltsoziologie. Sonderheft 36 der Kölner Zeitschrift für Soziologie und Sozialpsychologie. Opladen: Westdeutscher Verlag.

Dunlap, R. E. / Mertig, A. G., 1996: Weltweites Umweltbewusstsein. Eine Herausforderung für die sozialwissenschaftliche Theorie. 195-218 in A. Diekmann / C.C. Jaeger (Hrsg.): Umweltsoziologie. Sonderheft 36 der Kölner Zeitschrift für Soziologie und Sozialpsychologie. Opladen: Westdeutscher Verlag.

Dunlap, R.E. / Gallup, G.H. / Gallup, A.M., 1993: Of Global Concern: Results of the Health of the Planet Survey. Environment 35: 7-15, 33-39.

Europäische Gemeinschaften, 1995-2005a: Beitrittstrategien für die Umwelt, http://europa.eu.int/scadplus/leg/de/lvb/128057.htm (Download 06.07.2005).

Europäische Gemeinschaften, 1995-2000b: Umwelt: Zusammenarbeit mit Drittländern/ [Land] - Die Übernahme des gemeinschaftlichen Besitzstandes, http://europa.eu.int/scadplus/leg/de/s15011.htm (Download 15.07.2005). (Berichte für die einzelnen Länder).

European Commission, 2005: Special Eurobarometer 217 "Attitudes of European citizens towards environment", http://europa.eu.int/comm/public_opinion/archives/ebs/ebs_217_en.pdf (Download 23.8.05).

Fehr, E. / Fischbacher, U., 2002: Why Social Preferences Matter - The Impact of Non-selfish Motives on Competition, Cooperation and Incentives. The Economic Journal 112: C1-C33.

Fligstein, N. / Stone Sweet, A., 2002: Constructing Polities and Markets. An Institutionalist Account of European Integration. American Journal of Sociology 107: 1206-1243.

Franzen, A. / Meyer, R., 2004: Klimawandel des Umweltbewusstseins? Eine Analyse des ISSP 2000. Zeitschrift für Soziologie 33: 119-137.

Franzen, A., 2003: Environmental Attitudes in International Comparison: An Analysis of the ISSP Surveys 1993 and 2000. Social Science Quarterly 84: 297-308.

Fuchs, D. / Klingemann, H.-D., 1990: The Left-Right Schema. S. 203-234 in: M.K. Jennings / J. van Deth et al. (Hrsg.): Continuities in Political Action - A Longitudinal Study of Political Orientations in Three Western Democracies, Berlin/New York: Walter de Gruyter. 
Fürst, V., 2004: Umweltpolitisches Regieren im Wandel. Zur Transformation von Staatlichkeit in weltmarktoffenen Staaten. Diss., Berlin: Freie Universität Berlin (http://www.diss.fuberlin.de/2004/192/).

Gerhards, J. / Hölscher, M., 2003: Kulturelle Unterschiede zwischen Mitglieds- und Beitrittsländern der EU. Das Beispiel Familien- und Gleichberechtigungsvorstellungen. Zeitschrift für Soziologie 32: 206-225.

Gerhards, J., unter Mitarbeit von M. Hölscher, 2005: Kulturelle Unterschiede in der europäischen Union. Ein Vergleich zwischen Mitgliedsländern, Beitrittskandidaten und der Türkei. Wiesbaden: VS.

Greenbaum, A., 1995: Taking Stock of Two Decades of Research on the Social Basis of Environmental Concern. S. 125-152 in: M.D. Metha / E. Quellet (Hrsg.): Environmental Sociology. North York: Captus Press.

Huber, P.J., 1967: The Behavior of Maximum Likelihood Estimates Under NonStandard Conditions. S. 221-233 in: L.M. LeCam / J. Neyman (Hrsg.): Proceedings of the Fifth Annual Berkeley Symposium on Mathematical Statistics and Probability. Vol. I, Berkeley, Ca.: University of California Press.

Inglehart, R., 1971: The Silent Revolution in Europe. American Political Science Review 65: 991-1017.

Inglehart, R., 1983: Traditionelle politische Trennungslinien und die Entwicklung der neuen Politik in westlichen Gesellschaften. Politische Vierteljahresschrift 24: 139-165.

Inglehart, R., 1989: Kultureller Umbruch. Frankfurt/M.: Campus.

Inglehart, R., 1995: Public Support for the Environmental Protection: Objective Problems and Subjective Values in 43 Societies. PS: Political Science and Politics 28: 57-72.

Inglehart, R., 1997: Modernization and Postmodernization. Cultural, Economic and Political Change in 43 Societies. Princeton: Princeton University Press.

Johnson, S. / Corcelle, G., 1989: The Environmental Policy of the European Communities. London: Graham \& Trotman.

Knill, C., 2001: The Europeanisation of National Administrations. Patterns of Institutional Change and Persistence. Cambridge: Cambridge University Press.

Knill, C., 2003: Europäische Umweltpolitik. Steuerungsprobleme und Regulierungsmuster im Mehrebenensystem. Opladen: Leske \& Budrich.

Kohler-Koch, B. / Conzelmann, Th. /Knodt, M., 2004: Europäische Integration - Europäisches Regieren. Wiesbaden: VS.

Kohn, M.L. / Schooler, C., 1982: Job Conditions and Personality. American Sociological Review 87: 1257-1286.

Krämer, L., 1992: Focus on European Environmental Law. London: Sweet \& Maxwell.

Langeheine, R. / Lehmann, J., 1986: Ein neuer Blick auf die soziale Basis des Umweltbewusstseins. Zeitschrift für Soziologie 15: 378-384.

Lepsius, M.R., 1990: Der europäische Nationalstaat: Erbe oder Zukunft. S. 256-268 in: ders. (Hrsg.): Interessen, Ideen und Institutionen. Opladen: Westdeutscher Verlag.

McCormick, J., 1999: Environmental Policy. S. 193-210 in: L. Cram / D. Dinan / N. Nugent (Hrsg.): Developments in the European Union. Basingstoke, London: Macmillan.

Nunner-Winkler, G., 2000: Wandel in den Moralvorstellungen: Ein Generationenvergleich. S. 299-336 in: W. Edelstein / G. Nunner-Winkler (Hrsg.): Moral im sozialen Kontext. Frankfurt a.M.: Suhrkamp.

Page, B. I. / Shapiro, R. Y., 1983: Effects of Public Opinion on Policy. American Political Science Review 77: 175-190.

Preisendörfer, P., 1999: Umwelteinstellungen und Umweltverhalten in Deutschland. Empirische Befunde und Analysen auf der Grundlage der Bevölkerungsumfragen „Umweltbewußtsein in Deutschland 1991 - 1998. Opladen: Leske \& Budrich.

Preisendörfer, P. / Franzen, A., 1996: Der schöne Schein des Umweltbewußtseins. Zu den Ursachen und Konsequenzen von Umwelteinstellungen in der Bevölkerung. S. 219-244 in: A. Diekmann / 
C.C. Jaeger (Hrsg.): Umweltsoziologie. Sonderheft 36 der Kölner Zeitschrift für Soziologie und Sozialpsychologie. Opladen: Westdeutscher Verlag.

Roose, J., 2003: Die Europäisierung der Umweltbewegung. Umweltorganisationen auf dem langen Weg nach Brüssel. Wiesbaden: Westdeutscher Verlag.

Roth-Behrendt, D. / Nowak, A. 2004: Die Umweltpolitik der europäischen Union. S. 305-322 in: W. Weidenfeld (Hrsg.): Die europäische Union. Politisches System und Politikbereiche. Bonn: Bundeszentrale für politische Bildung.

Snow, D.A. / Benford, R.D., 1988: Ideology, Frame Resonance, and Participant Mobilization. S. 197-217 in: B. Klandermans / H. Kriesi / S. Tarrow (Hrsg.): International Social Movement Research 1. Greenwich, Connecticut: JAI Press.

Snow, D.A. / Rochford, E.B. / Worden, S.K. / Benford, R.D., 1986: Frame Alignment Processes, Micromobilization, and Movement Participation. American Sociological Review 51: 464-481.

Stone Sweet, A. / Sandtholtz, W. / Fligstein, N. (Hrsg.), 2001: The Institutionlization of Europe. Oxford: Oxford University Press.

Weidenfeld, W. (Hrsg.), 2004: Die europäische Union. Politisches System und Politikbereiche. Bundeszentrale für politische Bildung: Bonn.

Wegener, B., 2001: Solidarity, Justice, and Social Change: Germany's Ten Years of Unification. S. 207233 in: D. Pollack / J. Jacobs / O. Müller / G. Pickel (Hrsg.): Political Culture in Central and Eastern Europe. Ashgate: Aldershot.

Wessels, W., 1997: An Ever Closer Fusion? A Dynamic Macropolitical View on Integration Processes. Journal of Common Market Studies 35: 267-299. 
Anhang 1: Kriterien zur Übernahme des gemeinschaftlichen Besitzstandes der EU auf dem Gebiet des Umweltrechts (Quelle: Europäische Gemeinschaften 1995-2000b)

(1) Integration von Umweltbelangen in andere Politikbereiche;

(2) Grad der Übereinstimmung der horizontalen umweltrechtlichen Vorschriften eines Landes mit dem Umwelt-Acquis (u.a. Ratifizierung des Kyoto-Protokolls und Unterzeichnung anderer Abkommen);

(3) Wasserqualität (Schutz des Grundwassers, Schutz der Gewässer vor Verunreinigung durch Nitrate und gefährliche Stoffe, Verminderung des Fluorgehalts im Trinkwasser, Abwasserbehandlung, Ableitung gefährlicher Stoffe);

(4) Abfallwirtschaft (Entsorgung von Abfällen, Verpackungen und Altfahrzeugen, Verwertung und Beseitigung gefährlicher Abfälle und Verpackungen, Verpackungssteuern, Aufbau eines Netzes von Abfallbeseitigungsanlagen und eines Genehmigungssystems);

(5) Kontrolle der Verschmutzung durch Industrieanlagen und Risikomanagement (z.B. Emission flüchtiger organischer Verbindungen, die bei der Verwendung von Lösungsmitteln entstehen, Abfallverbrennung, Großfeuerungsanlagen, Festlegung nationaler Emissionshöchstmengen, Integrierte Vermeidung und Verminderung der Umweltverschmutzung);

(6) Luftqualität (z.B. Emissionsraten und -abgaben für luftverunreinigende Stoffe, Genfer Übereinkommen über weiträumige grenzüberschreitende Luftverunreinigung, Ratifizierung der Londoner und Kopenhagener Änderungen zum Protokoll von Montreal);

(7) Chemikalien (Bewertung und Überwachung der Risiken chemischer Altstoffe, Kenzeichnungs-, Verpackungs-, Klassifizierungs-, Identifizierungs- und Notifizierungsverfahren, Einfuhr- und Ausfuhrregelungen für bestimmte gefährliche Stoffe);

(8) Genetisch veränderte Organismen (GVO);

(9) Strahlenschutz und nukleare Sicherheit (die nukleare Sicherheit wird im Kapitel „Energie" behandelt);

(10) Naturschutz (die Beitrittsländer mussten Listen von Gebieten erstellen, die sie als Gebiete von gemeinschaftlicher Bedeutung oder als besondere Schutzgebiete vorschlagen, Erlassen eines Naturdenkmälergesetzes, nationale Programme zur Verwirklichung von Natura 2000, Jagdbestimmungen);

(12) Lärmemission. 
Anhang 2: Variablenbeschreibung ISSP 2000 (nur rekodierte \& importierte Variablen)

\begin{tabular}{|c|c|c|c|}
\hline Variable & Range & Beschreibung & Quelle \\
\hline Familieneinkommen & $1,10-13,82$ & $\begin{array}{l}\text { Operationalisierung: logarith- } \\
\text { miert }\end{array}$ & \\
\hline Postmaterialismus & $0-2$ & $\begin{array}{l}\text { 4-Item-Ranking-Skala; Operatio- } \\
\text { nalisierung nach Inglehart (1990): } \\
\qquad \begin{aligned} 0 & =\text { kein postmat. Item, } \\
1 & =1 \text { postmat. Item, } \\
2 & =2 \text { postmat. Items. }\end{aligned}\end{array}$ & \\
\hline $\begin{array}{l}\text { GDP per Capita } \\
\text { (in Kaufkraftparitä- } \\
\text { ten) }\end{array}$ & $5071-25918$ & $\begin{array}{l}\text { Standard-Wohlstandsmaß } \\
\text { (HDI-Bericht 2001) }\end{array}$ & $\begin{array}{l}\text { http://hdr.undp.org/reports/ } \\
\text { global/2001/en/pdf/back.pdf }\end{array}$ \\
\hline $\begin{array}{l}\text { Umweltqualität } \\
\text { (ESI-Index) }\end{array}$ & $47,4-80,5$ & $\begin{array}{l}\text { Der Environmental Sustainability } \\
\text { Index (ESI) des „Environmental } \\
\text { Performance Measurement Pro- } \\
\text { ject“ (Univ. Yale) besteht aus } 22 \\
\text { sozioökonomischen, umweltbe- } \\
\text { zogenen und institutionellen In- } \\
\text { dikaktoren die die ökologische } \\
\text { Nachhaltigkeit eines Landes be- } \\
\text { einflussen (ESI-Bericht 2001) }\end{array}$ & http://www.yale.edu/esi/ \\
\hline
\end{tabular}




\section{Anhang 3: Kausalanalysen mit Faktorindex (ISSP 2000)}

Tabelle 4: $\quad$ Präferenzen zum Umweltschutz II (Faktorindex)

\begin{tabular}{|c|c|c|}
\hline & & $h^{2}$ \\
\hline $\begin{array}{l}\text { Für jemand wie mich ist es einfach schwierig, viel für } \\
\text { die Umwelt zu tun }\end{array}$ & 71770 & ,515 \\
\hline $\begin{array}{l}\text { Es gibt wichtigeres im Leben zu tun, als die Umwelt } \\
\text { zu schützen }\end{array}$ & 70107 & ,492 \\
\hline $\begin{array}{l}\text { Es ist zwecklos, meinen Beitrag für die Umwelt zu } \\
\text { leisten, solange andere sich nicht genauso verhalten }\end{array}$ & ,75221 & ,566 \\
\hline $\begin{array}{l}\text { Viele Behauptungen über die Gefährdung der Um- } \\
\text { welt sind übertrieben }\end{array}$ & 68933 & ,475 \\
\hline Eigenwert & 2,048 & $51,2 \%$ \\
\hline
\end{tabular}

Quelle: ISSP 2000; N = 5349; Hauptkomponentenanalyse.

Tabelle 5: Determinanten von Präferenzen für Umweltschutz (Faktorindex)

\begin{tabular}{lccc}
\hline & Modell 1 & Modell 2 & Modell 3 \\
\hline SoziodemografischeMerkmale &,$- 126^{* * *}$ &,$- 115^{* * *}$ &,$- 116^{* * *}$ \\
Alter (in Jahren) & $(-10,68)$ & $(-7,78)$ & $(-7,28)$ \\
Familieneinkommen &, 017 &, 013 &,- 018 \\
& $(0,14)$ & $(0,12)$ & $(-0,25)$ \\
Klassenlage &, $145^{* * *}$ &, $142^{* * *}$ &, $088^{* * *}$ \\
GeneralisierteEinstellungen & $(5,00)$ & $(6,15)$ & $(6,38)$ \\
Politische & &,$- 114^{* *}$ &,$- 078^{*}$ \\
Selbsteinstufung & & $(-2,94)$ & $(-2,72)$ \\
Postmaterialismus &, $170^{* * *}$ &, $127^{* * *}$ \\
(Inglehart-Index) & $(6,20)$ & $(9,89)$ \\
\hline Ländermerkmale & &, 241 \\
GDP per capita (PPP) & & $(2,83)^{*}$ \\
Umweltqualität & &, $091^{* *}$ \\
(ESI-Index) & & $(3,24)$ \\
\hline R ${ }^{2}$ & &, $\mathbf{1 7 2}$ \\
\hline
\end{tabular}

Quelle: ISSP 2000; N =5349; hierarchische Regressionsmodelle mit robusten Standardfehlern unter Berücksichtigung von Clustern nach Länderzugehörigkeit; angegeben sind standardisierte Regressionskoeffizienten; $\mathrm{t}$-Werte in Klammern; ${ }^{*} \mathrm{p}_{\mathrm{t}}<.05,{ }^{* *} \mathrm{p}_{\mathrm{t}}<.01,{ }^{* * *} \mathrm{p}_{\mathrm{t}}<.001$. 